\title{
Proposta de um sistema de monitoramento de incêndios ambientais utilizando o ESP32-CAM
}

\author{
João Pedro Cunha dos Santos ${ }^{1,3}$, Guilherme Henrique Alves da Silva ${ }^{1,3}$, Marco \\ Antonio Franzin Leite ${ }^{1}$, Juliana Saragiotto Silva ${ }^{2}$
}

${ }^{1}$ Departamento de Engenharia Elétrica e Automação - Campus Cuiabá - Cel.

Octayde Jorge da Silva - Instituto Federal de Mato Grosso - R. Zulmira Canavarros, 95 - Centro, Cuiabá - MT, 78005-390 - Cuiabá-MT - Brasil.

${ }^{2}$ Departamento de Computação - Campus Cuiabá - Cel. Octayde Jorge da Silva Instituto Federal de Mato Grosso - Cuiabá-MT - Brasil

${ }^{3}$ Programa de Educação Tutorial AutoNet, Instituto Federal do Mato Grosso Campus Cuiabá - Cel. Octayde Jorge da Silva - Cuiabá - MT, Brasil

joaopedrocunhastolo@gmail.com, guilhermehenriquee9@gmail.com,
marcofranzin1999@gmail.com, juliana.silva@cba.ifmt.edu.br

Abstract. The planet has been undergoing more rapid changes in its biosphere, such as the extinction of biomes. One of the main causes of these changes is the emission of pollutant gases into the atmosphere, such as carbon dioxide. In this context, the aim of this work is to present a low-cost proposal for the development of a support system for environmental firefighting, from the monitoring of images using the ESP32-CAM. For the development of this work, the following steps were taken: (i) literature review; (ii) analysis of the prototype requirements; and (iii) identification of application areas and mapping of future research for the creation of the prototype. As a result, it was possible to detail all the necessary elements for the construction of the prototype.

Resumo. O planeta vem sofrendo mudanças de forma mais acelerada em sua biosfera, como a extinção de biomas. Um dos principais causadores dessas mudanças é a emissão de gases poluentes na atmosfera, como o gás carbônico. Diante desse contexto, o objetivo do trabalho é apresentar uma proposta de baixo custo para o desenvolvimento de um sistema de apoio ao combate de incêndios ambientais, a partir do monitoramento de imagens utilizando o ESP32-CAM. Para o desenvolvimento desse trabalho foram realizadas as seguintes etapas: (i) revisão bibliográfica; (ii) análise dos requisitos do protótipo; e (iii) identificação das áreas de aplicação e mapeamento de futuras pesquisas para a criação do protótipo. Como resultado, foi possível detalhar todos os elementos necessários para a construção do protótipo.

\section{Introdução}

O meio ambiente vem sendo o tema central abordado em diversas conferências promovidas pelas Nações Unidas, por exemplo, a Conferência de Estocolmo (em 1972), a ECO-92, a RIO +10 e a RIO +20, culminando, em 2015, o acordo de Paris que buscava mobilizar a diminuição de lançamento de gases causadores do efeito estufa na atmosfera [Silva 2013]. As queimadas são uma das grandes responsáveis 
pelo aquecimento global, que tanto preocupa a comunidade científica, pois nos últimos 250 anos a temperatura da terra aumentou 1,5 graus [Artaxo 2013].

$\mathrm{O}$ aquecimento global trata do aumento gradual de temperatura do planeta Terra. Entretanto, nos últimos anos, esse aumento tem acontecido de maneira desregulada. Essa elevação de temperatura da terra tem colaborado com o derretimento das geleiras e isso tem causado o aumento de volume de água dos oceanos. Além disso, o aquecimento global causa a elevação de temperatura das águas, que, por sua vez, contribuem para a diminuição da movimentação das correntes marítimas, prejudicando a migração de várias espécies e os ecossistemas dos oceanos. [Molion 2005]

Outro fator que tem contribuído para o aquecimento global é o aumento considerável dos incêndios ambientais, especialmente na região Centro-Oeste do Brasil. Isso pode ser verificado no site do Instituto Nacional de Pesquisas Espaciais (INPE), que tem registrado e monitorado, desde 1998, os focos ativos de incêndios que ocorrem no país, por estado, região ou bioma. De acordo com esse banco de dados, 2007 foi o ano com o maior número de focos de queimadas, com quase 138 mil casos registrados. [Inpe 2021]

É justamente neste contexto, que existe uma oportunidade de pesquisa para o desenvolvimento de propostas que se utilizem das Tecnologias de Informação e Comunicação, para apoiar ações no combate ou mitigação de fatores que contribuem para a propagação de incêndios e, assim, promover o cuidado com o meio ambiente. Esta área é comumente descrita como Informática para a Biodiversidade.

Assim, o objetivo deste trabalho é propor um sistema de monitoramento de incêndios ambientais, de baixo custo, utilizando o ESP32-CAM.

Desta forma, este artigo está estruturado em 5 seções, incluindo esta introdução. A seção 2 apresenta os conceitos básicos sobre a temática em estudo. $\mathrm{Na}$ sequência, a seção 3 discorre sobre a metodologia utilizada no desenvolvimento do artigo. Já, a seção 4, descreve os resultados do trabalho - detalhando os elementos necessários ao desenvolvimento do protótipo. Por fim, a seção 5, apresenta as conclusões do trabalho.

\section{Conceitos básicos}

Esta seção tem por objetivo descrever, de forma sucinta, os termos fundamentais para a compreensão desta pesquisa, como incêndios ambientais, microcontroladores e sensores.

\subsection{Incêndios Ambientais}

Os incêndios ambientais são perturbações causadas pelo fogo, de maneira descontrolada, que ocorrem, geralmente, em áreas rurais ou em biomas [Castro et al. 2002]. Dependendo das condições do ambiente, os incêndios possuem a capacidade de se alastrar de forma muito rápida. Outro aspecto observado é que grande parte dos incêndios são causados por seres humanos; no entanto, também podem ser motivados por fenômenos naturais, como raios elétricos, reações químicas ou concentração de raios solares [Guimarães et al. 2014].

Dentre os registros mapeados pelo INPE estima-se que, em agosto de 2019, o fogo devastou uma área de 29,944 $\mathrm{km}^{2}$ da Amazônia; e, em 2010, foi registrado o pior incêndio florestal na Amazônia, cobrindo uma área de 43,178 km² [Inpe 2021]. 
Para a identificação desses incêndios, o INPE se utiliza de diversos recursos tecnológicos, como o uso de sensores e outros dispositivos de hardware que possibilitam a captura de imagens e variáveis ambientais. No entanto, o uso de tecnologias para esta finalidade requer um investimento de alto custo que, muitas vezes, o pequeno agricultor não dispõe. Assim, uma alternativa seria o uso de dispositivos de baixo custo, para o desenvolvimento de sistemas de apoio a esses agricultores, utilizando-se de microcontroladores como o ESP32 - que é descrito na seção a seguir.

\subsection{Microcontroladores}

Os microcontroladores são circuitos integrados, que consistem na junção entre componentes de hardware e recursos de software, na qual podem executar milhares de funções, desde que seja programado previamente. [Oliveira Júnior e Duarte 2010].

Em função disso, são considerados como um microcomputador, pois unem, em um único chip, as funcionalidades de vários periféricos, como: um processador em formato de Unidade Lógica e Aritmética (ULA), memória, contadores, registradores, conversores de entradas e saídas, temporizadores e dispositivos de comunicação serial; alguns processadores mais avançados possuem, inclusive, comunicação por rádio frequência e por bluetooth, além de wifi. [Pereira 2007]

$\mathrm{Na}$ atualidade, há alguns modelos de microcontroladores no mercado, como: Programmable Interface Controller (PIC), Arduino, Raspberry Pi, Accessory Development Kit (ADK) e ESP. Cada um possui as suas particularidades e pode ser escolhido conforme a necessidade do pesquisador. A diferença entre eles está em seus módulos periféricos, como: conversores analógicos-digitais $(A / D)$, módulos de controle de largura de pulso (Pulse-Width Control, $P W M$ ) e comunicações seriais [Ordonez 2005].

Para este trabalho foi escolhido o kit de desenvolvimento ESP32-CAM, que deriva da família do microcontrolador ESP. A escolha ocorreu em função deste microcontrolador dispor de recursos como o wifi integrado, uma câmera com diodo emissor de luz (Ligth Emitting Diode, LED - que vem juntamente com o módulo do ESP32), um processador mais robusto em relação aos outros, um slot para cartão micro-SD e uma antena externa (com o propósito de aumentar o alcance de comunicação). Desta forma, torna-se possível a comunicação com os sensores, para que a coleta de dados seja realizada - como descrito a seguir.

\subsection{Sensores}

O sensor é um dispositivo usado para mensurar variáveis físicas ou químicas. Tem como função a captação de um estímulo ou um sinal natural do ambiente. Este sinal capturado pode ser utilizado para diversas finalidades, como monitoramento ou armazenamento de dados. [Thomazini 2005]

Um exemplo desse tipo de aplicação são os sensores utilizados para a coleta de dados de variáveis ambientais, como temperatura, umidade e concentração de gás carbônico $\left(\mathrm{CO}_{2}\right)$. Desta forma, podem apoiar ações de monitoramento no combate e prevenção de incêndios ambientais - como disposto na seção Resultados.

Uma vez que, alguns conceitos essenciais do trabalho foram apresentados, a próxima seção visa explicitar os materiais e métodos utilizados para o desenvolvimento da proposta. 


\section{Materiais e métodos}

O trabalho consistiu em uma proposta de implementação de um sistema de combate a incêndios ambientais, de baixo custo, utilizando o módulo ESP32-CAM e alguns sensores para o monitoramento e envio de dados de variáveis como temperatura, umidade e concentração de gás carbônico.

Deste modo, o desenvolvimento desta pesquisa compreendeu as seguintes etapas: (i) revisão bibliográfica; (ii) análise dos requisitos do protótipo; (iii) identificação das áreas de aplicação e mapeamento de futuras pesquisas para a montagem do protótipo.

$\mathrm{Na}$ etapa (i), foram utilizadas como apoio as ferramentas de busca Google Acadêmico, Biblioteca Digital Brasileira de Teses e Dissertações (BDTD) e Portal de Periódicos da Coordenação de Aperfeiçoamento de Pessoal de Nível Superior (CAPES), a partir das seguintes palavras-chave: "Microcontroladores", "Robótica contra incêndios" e "Tecnologia no combate contra incêndios". Foram mapeados trabalhos publicados entre 2003 e 2021.

$\mathrm{Na}$ etapa (ii), foram levantados os requisitos para a construção do protótipo, obtendo-se a lista dos seguintes recursos de hardware: kit de desenvolvimento integrado ao microcontrolador, o ESP32-CAM - Figura 1a (para o armazenamento e o processamento de dados), juntamente com os seguintes sensores: de gás - $M Q 2-$ Figura $1 \mathrm{~b}$ (para a coleta do nível de concentração de $\mathrm{CO}_{2}$ ); de temperatura e umidade - DHT22 - Figura 1c (para a coleta da temperatura e da umidade do ambiente). Igualmente, para a alimentação dos circuitos devem ser utilizadas placas solares de $12 \mathrm{~V}$ e uma fonte simétrica ${ }^{1}$ de $12 \mathrm{~V}$, que possa distribuir $3,3 \mathrm{~V}-5 \mathrm{~V}$. E, também, para o envio e recebimento de dados utiliza-se uma rede de longo alcance (Long Range$L o R a)$.

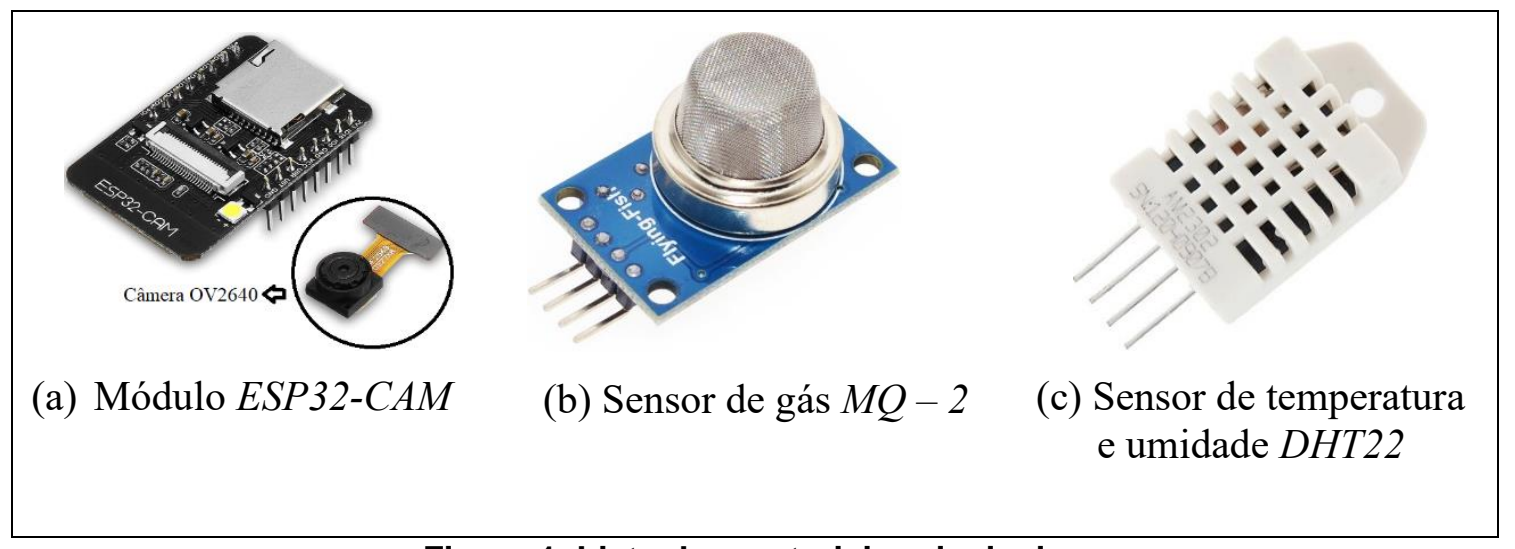

Figura 1. Lista dos materiais principais

Após o mapeamento dos requisitos necessários para a construção do protótipo, a última etapa (iii) consistiu na identificação de áreas mais propensas ao aparecimento de focos de incêndios, bem como perspectivas de trabalhos futuros.

\section{Resultados}

Esta seção tem como finalidade apresentar a proposta do protótipo que foi idealizado nesta pesquisa, de forma que descreva os principais equipamentos e o princípio de funcionamento do sistema.

\footnotetext{
${ }^{1}$ São fontes de alimentação que geram sinais de tensão positivos e negativos.
} 


\subsection{Sobre os componentes de hardware e de software do protótipo}

A ideia do protótipo consiste em uma central que possa fazer o monitoramento, alarme de focos e combate de incêndios. Desta forma, para a montagem do protótipo será utilizado o ESP32-CAM, que tem como função realizar o processamento das informações coletadas pelos sensores, armazenamento de dados e execução de comandos já programados. Além disso, a câmera do ESP32-CAM será utilizada para a captação de imagens, em tempo real, das áreas inspecionadas.

O ESP32-CAM possui um grande diferencial, por possibilitar a comunicação sem fio (wireless) com outro dispositivo, seja por meio do bluetooth ou do wifi integrados ao microcontrolador. Outra característica que chama a atenção é o fato dele possuir uma câmera com flash embutido, que permite o armazenamento e o envio de imagens, em tempo real. Ademais, a sua programação pode ser feita através do ambiente de desenvolvimento integrado (Integrated Development Environment IDE) do Arduino [Kurniavawan 2019], que leva a vantagem por possuir um ambiente de programação agradável e simples.

Uma vez que, se tenha conhecimento dos recursos do microcontrolador a serem utilizados, na construção desta proposta, faz-se necessário, a partir de agora, entender como os sensores serão incorporados a este sistema.

Para a captura das variáveis temperatura e umidade, deve ser utilizado o sensor DHT22, que é a evolução do sensor DHT11. E, por isso, permite medições mais precisas do que o anterior, como a captura de valores de temperatura na faixa de -40 a +80 Celsius e, de umidade, entre 0 e 100\% [Marian 2021].

Com relação ao sensor para capturar moléculas de gás $\mathrm{CO}_{2}$ presentes no ambiente, será utilizado o $M Q-2$, que permitirá o envio desses dados, na forma de sinal, ao microcontrolador. O sensor $M Q-2$ é capaz detectar vários tipos de gases que estiverem na atmosfera local, como: butano, propano, metano, hidrogênio, álcool, gás natural ou, até mesmo, fumaça. [Poluolu 2021].

Já o procedimento de envio e recebimento de dados, entre a central de combate a incêndios e o protótipo (que ficará localizado em áreas ambientais), será realizado por meio da rede de longo alcance $\operatorname{LoR} a$, visto que, em áreas rurais, a distância entre esses pontos de conexão pode chegar à $15 \mathrm{~km}$ [Teixeira e Almeida 2014].

No que tange ao desenvolvimento do sistema, a parte de programação para a integração do microcontrolador com os sensores e o ESP32-CAM, pode ser realizada por meio da $I D E$ do Arduino, a partir da inclusão das bibliotecas específicas de cada componente. Em relação ao algoritmo de processamento digital de imagens, deve-se utilizar a plataforma Tensorflow, que é uma plataforma de Machine Learning, na qual se realiza a programação para o reconhecimento para focos de incêndios.

\subsection{Sobre o funcionamento do protótipo}

Quando os sensores captam os sinais no ambiente, eles enviam para a central de processamento do ESP32-CAM, com base nos dados coletados será feito a análise das variáveis de temperatura, umidade e gás, para posteriormente classificar o risco de incêndio naquela área. No que concerne, ao funcionamento do ESP32-CAM, quando a câmera percebe uma variação de pixel, por meio da sua lente, os dados dos pixels serão enviados para a central de processamento. 
$\mathrm{Na}$ sequência, de acordo com a programação implementada no microcontrolador (que consiste em avaliar os pixels que compõem a imagem), ele deve realizar o processamento das imagens recebidas no local, com o objetivo de identificar se são possíveis focos de incêndios ou não. Em caso afirmativo, enviam as informações para a central de combate a incêndios, por meio dos gateways $L o R a$, que encaminham os pacotes para servidor central de rede, emitindo um alerta.

É importante mencionar, ainda que, para a economia de energia, pode ser utilizada a função Modem sleep do ESP32-CAM, que permite otimizar o consumo de energia do microprocessador, mesmo estando em funcionamento contínuo. Esta função consiste na suspensão das atividades parciais do microprocessador, por um período de tempo. Desta forma, a comunicação por radiofrequência $L o R a$, que está integrada ao ESP32-CAM fica desligada e, assim que houver a necessidade de enviar algum dado, ela é habilitada novamente.

Face ao exposto, nota-se que o sistema proposto pode ser uma alternativa para analisar aspectos que indicam um foco de incêndio (imagens, concentração de $\mathrm{CO}_{2}$, índices de umidade e temperatura) e reportar para uma central, indicando, de forma rápida, o local afetado pelo foco. $\mathrm{Na}$ literatura, pode-se encontrar alguns artigos que tratam de assuntos semelhantes, como o trabalho de Pessin et al. (2007), que utilizou um sistema multi-agentes para a identificação de incêndios florestais, sem intervenção humana, por meio de um ambiente virtual de simulação realística. Neste sistema, utilizaram como objeto de pesquisa: sensores e a programação dos agentes.

Todavia, o diferencial desta proposta é a utilização de microcontroladores de baixo custo, a captação de imagens e de dados mensurados pelos sensores (em tempo real), para a análise, o armazenamento e o apoio à tomada de decisão.

\section{Conclusão}

Este trabalho teve como objetivo apresentar a proposta de um sistema, que auxiliasse no combate aos incêndios ambientais, apresentando todos os conhecimentos básicos, métodos e componentes essenciais para a confecção do protótipo. Apesar de ter sido encontrado poucos trabalhos relacionados ao tema, ainda assim, é um assunto pertinente, porque envolve uma proposta de baixo-custo, que pode trazer contribuições ao pequeno agricultar, no cuidado com o meio ambiente e com o futuro do planeta Terra.

Como perspectiva de trabalhos futuros, a ideia é realizar a montagem do protótipo, de forma completa, para realizar os testes necessários com os componentes propostos e, por conseguinte, obter conclusões em um estudo de caso real. Outra estratégia será a implementação de painéis solares, como forma de aproveitar a energia solar, que prevalece durante o dia. Além disso, pretende-se adicionar um banco de dados, na central de monitoramento do sistema, para que seja possível armazenar os dados coletados do ano e, assim, realizar uma análise histórica dos dados, com o intuito de prever novos incêndios ambientais, antes que eles aconteçam.

\section{Referências}

Artaxo, P. (2014) “Mudanças climáticas e o Brasil”. Revista USP, n. 103, p.8-12. 
Castro, F. C. Serra, G. Parola, J. Reis, J. Lourenço, L. Correia, S. (2003) Combate a incêndios florestais. 2. ed. Sintra: Escola Nacional de bombeiros. 94p.

Coutinho, M, L. (2016) Biomas Brasileiros. 1, ed. São Paulo: Oficina de Textos.

Guimarães, P. P.; Souza, M. S.; Fiedler, C. N.; Silva, G. A. "Análise dos impactos ambientais de um incêndio florestal". (2014) AGRARIAN ACADEMY, Centro científico conhecer. Goiânia, v.1, n.1, p.38-60, jan./abr. Disponível em: http://www.conhecer.org.br/Agrarian\%20Academy/2014a/analise $\% 20 \mathrm{dos} \% 20 \mathrm{imp}$ actos.pdf. Acesso em: 15 mar. 2021.

INPE. INSTITUTO NACIONAL DE PESQUISAS ESPACIAIS. (2021) "Programa Queimadas". Disponível em: https:/queimadas.dgi.inpe.br/queimadas/portalstatic/estatisticas_estados/. Acesso em: 16 out. 2021.

Kurniawan, A. (2019) Internet of Things: Projects with ESP32, Editora Packt Publishing, $1^{\text {a }}$ Edição, Birmingham.

Marian, P. (2021) "Digital Relative Humidity \& Temperature Sensor AM2302/DHT22". Disponível em: https://www.electroschematics.com/am2302dht22-datasheet/. Acesso em: 15 out. 2021.

Molion, L. C. B. (2005) "Aquecimento global, El Niños, machas solares, vulcões e Oscilação Decadal do Pacífico". Climanálise, 8, agosto. Disponível em: http://climanalise.cptec.inpe.br/ rclimanl/revista. Acesso em: 14 jun. 2021.

Oliveira Júnior, M.; Duarte, R. O. (2010) Introdução ao Projeto com Microcontroladores e Programação de Periféricos. Universidade de Minas Gerais. (Apostila de Introdução ao Projeto com Microcontroladores e Programação de Periféricos).

Ordonez, E. D. M.; Penteado, C. G.; SILVA, A. C. R. (2005) Microcontroladores e FPGAs - Aplicações em automação. São Paulo: Novatec. 384p.

Pereira, F. (2003) Microcontroladores em PIC: Programação em C. 1, ed. São Paulo: Érica.

Pessin, G.; Osório, F.; Musse, S.; Nonemmacher, V.; Ferreira, S. S. (2007) "Utilizando Agentes Autônomos com Aprendizado para a Identificação e Combate de Incêndios em Áreas Florestais". In: Anais do VII Simpósio de informática do Planalto Médio (SIPM'07). Passo fundo, RS, Brasil: Universidade de Passo Fundo (UPF).

Pololu. (2021) "Datasheet: MQ-2 semiconductor sensor for combustible gas". Disponível em: https://www.pololu.com/file/0J309/MQ2.pdf. Acesso em: 10 out. 2021.

Silva Junior, R.; Ferreira, L. C. (2013) "Sustentabilidade na era das conferências sobre meio ambiente e desenvolvimento - um olhar para ecologia e economia". Ambiente e Sociedade. São Paulo, v. 16, n. 1, mar.

Teixeira, G. B.; Almeida, J. V. P. (2014) "Rede LoRa ${ }^{\circledR}$ e Protocolo LoRaWan ${ }^{\circledR}$ aplicados na agricultura de precisão no Brasil". 77 f. Monografia (Bacharel em Engenharia Eletrônica) - Curso de Engenharia Eletrônica, da Universidade Tecnológica do Paraná.

Thomazini, D.; Albuquerque, B. U. P. (2005) Sensores Industriais: Fundamentos e Aplicações. 8, ed. São Paulo:Érica. 